\title{
NON-EQUILIBRIUM TIME EVOLUTION IN QUANTUM FIELD THEORY
}

\author{
Christof WETTERICH*) \\ Institut für Theoretische Physik \\ Universität Heidelberg \\ Philosophenweg 16, D 69120 Heidelberg \\ C.Wetterich@thphys.uni-heidelberg.de
}

\begin{abstract}
The time development of equal-time correlation functions in quantum mechanics and quantum field theory is described by an exact evolution equation for generating functionals. This permits a comparison between classical and quantum evolution in non-equilibrium systems.
\end{abstract}

*) This work was performed in part at CERN, Geneva. 
In a statistical description of nature only expectation values or correlations are observable. The dynamical laws describe how a given (complete) set of correlation functions at some initial time $t_{0}$ has evolved at some later time $t$. In a very general context this constitutes a differential evolution equation for the correlation functions or their generating functionals. Different dynamical laws, i.e. the difference between a classical and a quantum description, manifest themselves only in different evolution equations. For quantum mechanics of a few degrees of freedom we are accustomed to use the Schrödinger equation for pure states and to evaluate time-dependent expectation values in an ensemble specified by a density matrix. The classical counterpart are Newton's equations with the ensemble described by a probability distribution for initial values. For many degrees of freedom a more direct formulation of the dynamics in terms of an evolution equation for generating functionals of correlation functions may be advantageous. Then one only treats with the relevant observable information and may avoid additional complication in intermediate steps of their computation. For classical equations of motion such an evolution equation has been established recently [1]. It is the purpose of this note to develop the counterpart for quantum mechanics and quantum field theory.

The resulting evolution equation in quantum field theory is a functional differential equation. Its practical use depends on the ability to find realistic truncations for the time-dependent effective action - the generating functional for the 1PI-Green functions. Nevertheless, already at the present formal stage a comparison between classical and quantum evolution equations sheds some light on current dynamical simulations of non-equilibrium quantum field theory problems within a classical approximation for the field equations. It also provides a systematic framework to compute quantum corrections to a classical evolution, as relevant, for example, for inflationary periods in early cosmology.

Consider a system with an arbitrary number of degrees of freedom described by conjugate operators $Q_{i}, P_{i}, i=1, \ldots n,\left[Q_{i}, P_{i}\right]=i \delta_{i j},\left[Q_{i}, Q_{j}\right]=\left[P_{i}, P_{j}\right]=0, \quad(\hbar \equiv 1)$. The Hamiltonian! is given by

$$
H=\frac{1}{2 m} P^{2}+V[Q],
$$

where $P^{2}=P_{i} P_{i}$ is a scalar product (summation of repeated indices is always implied) and $V[Q]=V\left(Q_{1} \ldots Q_{n}\right)$. In the Heisenberg picture the operators $Q$ and $P$ depend on time according to

$$
\dot{Q}_{i}=\frac{1}{m} P_{i}, \quad \dot{P}_{i}=-V_{i}[Q]
$$

(We use a notation $V_{i}=\frac{\partial V}{\partial Q_{i}}, V_{i j}=\frac{\partial^{2} V}{\partial Q_{i} \partial Q_{j}}$, etc.). Let us now define the generating functional

$$
Z[j, h, t]=\operatorname{Tr}\left(e^{j Q(t)+h P(t)} \rho\right)
$$

where $\rho$ is the density matrix which is time-independent in the Heisenberg picture. It is a function (or functional for $n \rightarrow \infty$ ) of the time-independent sources $j_{i}$ and $h_{i}$. The coefficients

\footnotetext{
${ }^{1}$ Systems with different masses $m_{i}$ can be brought to this form by simultaneous rescaling of $Q_{i}$ and $P_{i}-$ in fact, we could also use a scaling with $m=1$. Our formalism can be generalized for arbitrary $H[P, Q]$ but we restrict the discussion here to the most common form quadratic in the momenta.
} 
of its Taylor expansion

$$
Z[j, h, t]=\sum_{k=0}^{\infty} \sum_{\ell=0}^{\infty} \frac{1}{k ! \ell !} z_{q_{1} \ldots q_{k}, r_{1} \ldots r_{\ell}}^{(k, \ell)}(t) j_{q_{1}} \ldots j_{q_{k}} \quad h_{r_{1}} \ldots h_{r_{\ell}}
$$

are time-dependent symmetrized expectation values of correlation functions. For a one-component example $(n=1)$

$$
z^{(k, \ell)}(t)=\left\langle\left(Q^{k} P^{\ell}\right)_{s}(t)\right\rangle=\operatorname{Tr}\left\{\left(Q^{k}(t) P^{\ell}(t)\right)_{s} \rho\right\}
$$

the symmetrized ordering $(\quad)_{s}$ stands for an equally weighted sum over all $\frac{(k+\ell) !}{k ! \ell !}$ possibilities to form different chains of $k$ operators $Q$ and $\ell$ operators $P$, i.e.,

$$
\left(Q^{2} P^{2}\right)_{s}=\frac{1}{6}\left(Q^{2} P^{2}+Q P^{2} Q+P^{2} Q^{2}+P Q^{2} P+Q P Q P+P Q P Q\right)
$$

The generalization to arbitrary $n$ is obvious. Knowledge of $Z[j, h, t]$ contains the complete information about the system. Macroscopic quantities and thermodynamic functions can be expressed in terms of correlation functions [2].

The time-dependence of $Z$ obeys the evolution equation $\left(\partial_{t} Z\right.$ is the time derivative at fixed $j$ and $h$ )

$$
\partial_{t} Z=i \operatorname{Tr}\left(\left[H, e^{j Q+h P}\right] \rho\right)
$$

Our aim is to express the right-hand side in terms of $Z$ and its derivatives with respect to $j$ and $h$. In order to deal with the problem of ordering the non-commuting operators we use

$$
i\left[H, e^{j Q+h P}\right]=\frac{1}{m} j_{i} \frac{\partial}{\partial h_{i}} e^{j Q+h P}+\left.i V\left[\frac{\partial}{\partial \beta}\right]\left[e^{\beta Q}, e^{j Q+h P}\right]\right|_{\beta=0}
$$

and evaluate the commutator with the Campbell-Barker-Hausdorff formula

$$
\left[e^{\beta Q}, e^{j Q+h P}\right]=\left(e^{\frac{i}{2} \beta h}-e^{-\frac{i}{2} \beta h}\right) e^{(\beta+j) Q+j P}
$$

For each of the two contributions on the right-hand side, one can express $\frac{\partial}{\partial \beta}$ by an appropriate combination of $\frac{\partial}{\partial j}$ and $h$. This leads to

$$
i\left[V[Q], e^{j Q+h P}\right]=i\left(V\left[\frac{\partial}{\partial j}+\frac{i}{2} h\right]-V\left[\frac{\partial}{\partial j}-\frac{i}{2} h\right]\right) e^{j Q+h P}
$$

and (8), (10) can now be inserted in the trace (7). Our final result is a linear partial differential equation for the evolution of $Z$ with a simple structure

$$
\begin{aligned}
\partial_{t} Z & =\mathcal{L}^{(Z)} Z \\
\mathcal{L}^{(Z)} & =\frac{1}{m} j_{i} \frac{\partial}{\partial h_{i}}+i\left(V\left[\frac{\partial}{\partial j}+\frac{i}{2} h\right]-V\left[\frac{\partial}{\partial j}-\frac{i}{2} h\right]\right)
\end{aligned}
$$


For a comparison with the time evolution of $Z$ in classical statistics [1] we expand $V$ in powers of $h$

$$
\begin{aligned}
& i V\left[\frac{\partial}{\partial j}+\frac{i}{2} h\right]-i V\left[\frac{\partial}{\partial j}-\frac{i}{2} h\right]=-V_{i}\left[\frac{\partial}{\partial j}\right] h_{i}+\frac{1}{24} V_{i j k}\left[\frac{\partial}{\partial j}\right] h_{i} h_{j} h_{k} \\
& -\frac{1}{1920} V_{i j k \ell m}\left[\frac{\partial}{\partial j}\right] h_{i} h_{j} h_{k} h_{\ell} h_{m}+\ldots
\end{aligned}
$$

The first term in this expansion reproduces the classical evolution equation. This leads to the important observation that for linear equations of motion ( $H$ quadratic in $Q$ and $P$ ) there is no difference in the time evolution of correlation functions between quantum statistics and classical statistics! The higher order terms appearing for non-linear equations of motion can be viewed as quantum corrections to the classical evolution. Restoring $\hbar$, they involve powers $\hbar^{2}, \hbar^{4}$, etc. For $V$ containing only up to quartic terms the quantum correction $\Delta \mathcal{L}_{Q M}^{(Z)}$ reads explicitly $\left(V_{i j k} \equiv V_{i j k}[0]\right)$

$$
\begin{aligned}
\mathcal{L}^{(Z)} & =\mathcal{L}_{c l}^{(Z)}+\Delta \mathcal{L}_{Q M}^{(Z)} \\
\mathcal{L}_{c l}^{(Z)} & =\frac{1}{m} j_{i} \frac{\partial}{\partial h_{i}}-h_{i} V_{i}\left[\frac{\partial}{\partial j}\right] \\
\Delta \mathcal{L}_{Q M}^{(Z)} & =\frac{1}{24} V_{i j k} h_{i} h_{j} h_{k}+\frac{1}{24} V_{i j k \ell} h_{i} h_{j} h_{k} \frac{\partial}{\partial j_{\ell}}
\end{aligned}
$$

It may be instructive to consider two examples. We first take a single anharmonic oscillator with

$$
V(Q)=\frac{1}{2} m \omega^{2}\left(Q^{2}+\frac{4}{3} \sqrt{2 m \omega} \gamma Q^{3}+2 m \omega \delta Q^{4}\right.
$$

In terms of a complex source

$$
J=\frac{1}{\sqrt{2 m \omega}}(j+i m \omega h)
$$

which is conjugate to the creation operator $a^{\dagger}$ of the harmonic oscillator the evolution equation reads

$$
\begin{aligned}
\partial_{t} Z= & -i \omega\left\{J^{*} \frac{\partial}{\partial J^{*}}-J \frac{\partial}{\partial J}+\left(J^{*}-J\right)\left[\gamma\left(\frac{\partial}{\partial J^{*}}+\frac{\partial}{\partial J}\right)^{2}+\delta\left(\frac{\partial}{\partial J^{*}}+\frac{\partial}{\partial J}\right)^{3}\right]\right. \\
& \left.+\frac{\gamma}{12}\left(J^{*}-J\right)^{3}+\frac{\delta}{4}\left(J^{*}-J\right)^{3}\left(\frac{\partial}{\partial J^{*}}+\frac{\partial}{\partial J}\right)\right\} Z
\end{aligned}
$$

In our picture the state of the system at a given time $t$ is described by $Z[J, t]$. For the harmonic oscillator $(\gamma=\delta=0)$ the stationary states or fixed points of $Z\left(\partial_{t} Z=0\right)$ are exactly those for which all terms in $Z$ involve an equal number of powers of $J$ and $J^{*}$. They correspond to incoherent mixtures of eigenstates of the number operator $a^{\dagger} a$ or energy eigenstates. It is intriguing that due to the equivalence of the quantum and classical evolution for linear equations of motion the quantum mechanical energy eigenstates can also be viewed as classically 
stationary probability distributions for commuting coordinate and momentum variables. For $\gamma, \delta \neq 0$ these particular states are not stationary any more. We know, nevertheless, that Eq. (16) must admit an infinite number of fixed point solutions which correspond exactly to incoherent mixtures of energy eigenstates of the anharmonic oscillator. This follows ifrom the general observation that Eq. (]) is equivalent to

$$
\partial_{t} Z=-i \operatorname{Tr}\left(e^{j Q+h P}[H, \rho]\right)
$$

and $H(t)=H\left(t_{0}\right)$. Every $\rho$ which commutes with $H$ defines a stationary state. An important qualitative difference between the quantum and classical evolution equation can be easily seen if we neglect $\delta$. The quantum mechanical evolution equation for $\ln Z$ contains a "correction" term $\frac{\gamma}{12}\left(J^{*}-J\right)^{3}$ which acts as an additional constant "force" on the connected correlation function for $P^{3}$, i.e., $\Delta \partial_{t}<P^{3}>_{c}=\frac{1}{12} \omega \gamma(2 m \omega)^{3 / 2}$. A cubic potential is not bounded from below. The local minimum at the origin is separated from the unstable part by a barrier. A classically stable initial ensemble with all energies below the barrier becomes unstable in quantum mechanics due to tunneling, as reflected by the additional "force". The essential features of this effect are not changed if we restore stability by a small positive non-zero $\delta$. Once reexpressed in terms of real sources $j$ and $h$, Eq. (16) is a real partial linear differential equation for a function of three variables $Z(j, h, t)$. A linear combination of two solutions is again a solution. A numerical solution of this equation contains at once the information about the time evolution of all expectation values of arbitrary powers of $Q$ and $P$ ! In particular, it seems interesting to investigate the outcome for classically chaotic systems.

Our second example is a linear chain of oscillators (with mass $m$ scaled to one and $d=1$ )

$$
H=\sum_{i}\left\{\frac{1}{2} P_{i}^{2}+\frac{1}{2} \mu^{2} Q_{i}^{2}+\frac{1}{3} \nu a^{-d / 2} Q_{i}^{3}+\frac{1}{8} \lambda a^{-d} Q_{i}^{4}+\frac{1}{2 a^{2}}\left(Q_{i+1}-Q_{i}\right)^{2}\right\}
$$

Here $a$ is the distance between two oscillators on the chain and we take periodic boundary conditions with a fixed length $\Omega=\sum_{i=1}^{n} a$. The evolution equation reads

$$
\begin{aligned}
\partial_{t} Z= & \sum_{i}\left\{j_{i} \frac{\partial}{\partial h_{i}}+\frac{1}{a^{2}} h_{i}\left(\frac{\partial}{\partial j_{i+1}}-2 \frac{\partial}{\partial j_{i}}+\frac{\partial}{\partial j_{i-1}}\right)\right. \\
& -h_{i}\left(\mu^{2} \frac{\partial}{\partial j_{i}}+\nu a^{-d / 2}\left(\frac{\partial}{\partial j_{i}}\right)^{2}+\frac{1}{2} \lambda a^{-d}\left(\frac{\partial}{\partial j_{i}}\right)^{3}\right) \\
& \left.+\frac{1}{12} \nu a^{-d / 2} h_{i}^{3}+\frac{1}{8} \lambda a^{-d} h_{i}^{3} \frac{\partial}{\partial j_{i}}\right\} Z
\end{aligned}
$$

Again, the last two terms are the quantum corrections.

The transition to a field theory can be made by taking the limit $a \rightarrow 0$ while keeping a non-zero "volume" $\Omega$. With the replacements $a^{-d / 2} Q_{i} \rightarrow \tilde{\varphi}(x), \quad a^{-d / 2} P_{i}=\tilde{\pi}(x), \quad a^{d} \sum_{i} \rightarrow$ $\int d^{d} x, \quad a^{-(d / 2+1)}\left(Q_{i+1}-Q_{i}\right) \rightarrow \partial \tilde{\varphi} / \partial x_{i}$ - we generalize here to an arbitrary dimension $d-$ the Hamiltonian (18) becomes

$$
H=\int d^{d} x\left\{\frac{1}{2} \tilde{\pi}^{2}(x)+\frac{1}{2} \partial_{i} \tilde{\varphi}(x) \partial_{i} \tilde{\varphi}(x)+U(\tilde{\varphi}(x))\right\}
$$




$$
U(\tilde{\varphi}(x))=\frac{1}{2} \mu^{2} \tilde{\varphi}^{2}(x)+\frac{1}{3} \nu \tilde{\varphi}^{3}(x)+\frac{1}{8} \lambda \tilde{\varphi}^{4}(x)
$$

This is the Hamiltonian of a relativistic scalar field theory with field equations $\tilde{\pi}(x)=\dot{\tilde{\varphi}}(x)$, $\dot{\tilde{\pi}}=\ddot{\tilde{\varphi}}=\Delta \tilde{\varphi}-\partial U / \partial \tilde{\varphi}$. Since also the commutation relation $\left(a^{-d} \delta_{i j} \rightarrow \delta\left(x-x^{\prime}\right)\right)$

$$
\left[\tilde{\varphi}(x), \tilde{\pi}\left(x^{\prime}\right)\right]=i \delta\left(x-x^{\prime}\right)
$$

is covariant under Lorentz transformations, our system describes a Lorentz-invariant quantum field theory. Here $Z$ becomes a functional of the sources $j_{\varphi}(x), j_{\pi}(x)$ which are related to the sources in the discrete version by $a^{-d / 2} j_{i} \rightarrow j_{\varphi}(x), \quad a^{-d / 2} h_{i} \rightarrow j_{\pi}(x), \quad a^{-d / 2} \frac{\partial}{\partial j_{i}}=\frac{\delta}{\delta j_{\varphi}(x)}$ and $a^{-d / 2} \frac{\partial}{\partial h_{i}}=\frac{\delta}{\delta j_{\pi}(x)}$, i.e., $Z=\operatorname{Tr}\left\{\exp \left(\int d^{d} x\left[j_{\varphi}(x) \tilde{\varphi}(x)+j_{\pi}(x) \tilde{\pi}(x)\right]\right) \rho\right\}$. We finally arrive at the time evolution equation for a scalar quantum field theory

$$
\begin{aligned}
\partial_{t} Z\left[j_{\varphi}, j_{\pi}, t\right] & =\int d^{d} x\left\{j_{\varphi}(x) \frac{\delta}{\delta j_{\pi}(x)}+j_{\pi}(x) \Delta \frac{\delta}{\delta j_{\varphi}(x)}\right. \\
& \left.+i U\left(\frac{\delta}{\delta j_{\varphi}(x)}+\frac{i}{2} j_{\pi}(x)\right)-i U\left(\frac{\delta}{\delta j_{\varphi}(x)}-\frac{i}{2} j_{\pi}(x)\right)\right\} Z\left[j_{\varphi}, j_{\pi}, t\right]
\end{aligned}
$$

Its generalization to several scalar fields $\tilde{\varphi}_{a}(x)$ is straightforward - with $\tilde{\pi}^{2} \rightarrow \tilde{\pi}_{a} \tilde{\pi}_{a}, \partial_{i} \tilde{\varphi} \partial_{i} \tilde{\varphi} \rightarrow$ $\partial_{i} \tilde{\varphi}_{a} \partial_{i} \tilde{\varphi}_{a}$ in $H$ (12) and commutation relation $\left[\tilde{\varphi}_{a}(x), \tilde{\pi}_{b}\left(x^{\prime}\right)\right]=i \delta\left(x-x^{\prime}\right) \delta_{a b}$ we only have to replace $j_{\varphi} \frac{\delta}{\delta j_{\pi}} \rightarrow j_{\varphi_{a}} \frac{\delta}{\delta j_{\pi_{a}}}, j_{\pi} \Delta \frac{\delta}{\delta j_{\varphi}} \rightarrow j_{\pi_{a}} \Delta \frac{\delta}{\delta j_{\phi_{a}}}$ in Eq. (22). All symmetries of $U(\tilde{\varphi})$ are preserved by the evolution equation in the sense that an initially symmetric state remains so at later time. This does not preclude spontaneous symmetry breaking in the course of the evolution it may be detected by adding a small symmetry breaking linear term in $U$ or by starting with a slightly asymmetric initial state.

Equation (22) is a functional differential equation and its approximate solution has to proceed by some truncation. For this purpose it seems an advantage to switch to the generating functional for the 1PI-Green functions, $\Gamma[\varphi, \pi, t]=-\ln Z[j, t]+\int d^{d} x\left(j_{\varphi}(x) \varphi(x)+j_{\pi}(x) \pi(x)\right)$, where $\varphi(x)=\frac{\partial \ln Z}{\delta j_{\varphi}(x)}, \pi(x)=\frac{\delta \ln Z}{\delta j_{\pi}(x)}$. The derivation of the evolution equation for the effective action $\Gamma$ proceeds as in Ref. [1] and we only give here the result for the Hamiltonian (20) with $\partial_{t} \Gamma$ a time derivative at fixed $\varphi$ and $\pi$

$$
\begin{aligned}
\partial_{t} \Gamma= & -\left(\mathcal{L}_{c l}^{(\Gamma)}+\Delta \mathcal{L}_{Q M}^{(\Gamma)}\right) \Gamma \\
\mathcal{L}_{c l}^{(\Gamma)}= & \int d^{d} x\left\{\pi(x) \frac{\delta}{\delta \varphi(x)}+\varphi(x)\left(\Delta-\mu^{2}\right) \frac{\delta}{\delta \pi(x)}\right. \\
& -\left[\nu\left(\varphi^{2}(x)+G_{\varphi \varphi}(x, x)\right)+\frac{\lambda}{2}\left(\varphi^{3}(x)+3 \varphi(x) G_{\varphi \varphi}(x, x)\right.\right. \\
& -\int d x_{1} d x_{2} d x_{3} G_{\varphi \gamma_{1}}\left(x, x_{1}\right) G_{\varphi \gamma_{2}}\left(x, x_{2}\right) \\
& \left.\left.\left.G_{\varphi \gamma_{3}}\left(x, x_{3}\right) \frac{\delta^{3} \Gamma}{\delta \hat{\varphi}_{\gamma_{1}}\left(x_{1}\right) \delta \hat{\varphi}_{\gamma_{2}}\left(x_{2}\right) \delta \hat{\varphi}_{\gamma_{3}}\left(x_{3}\right)}\right)\right] \frac{\delta}{\delta \pi(x)}\right\} \\
\Delta \mathcal{L}_{Q M}^{(\Gamma)}= & \int d^{d} x\left\{\frac{\nu}{12}\left(\frac{\delta \Gamma}{\delta \pi(x)}\right)^{3}+\frac{\lambda}{8} \varphi(x)\left(\frac{\delta \Gamma}{\delta \pi(x)}\right)^{3}\right\}
\end{aligned}
$$


Here $G_{\gamma \gamma^{\prime}}\left(x, x^{\prime}\right)=\left\langle\hat{\tilde{\varphi}}_{\gamma}(x) \hat{\tilde{\varphi}}_{\gamma^{\prime}}\left(x^{\prime}\right)\right\rangle_{j}-\hat{\tilde{\varphi}}_{\gamma}(x) \hat{\tilde{\varphi}}_{\gamma^{\prime}}\left(x^{\prime}\right)$ is the propagator in presence of sources where $\hat{\varphi}_{\gamma}, \gamma=\varphi, \pi$ is a shorthand for $(\varphi, \pi)$. The propagator can in turn be expressed by the inverse of the matrix] of second functional derivatives of $\Gamma$, i.e., $G_{\gamma \gamma^{\prime}}\left(x, x^{\prime}\right)=\left(\Gamma^{(2)}\right)_{\gamma \gamma^{\prime}}^{-1}\left(x, x^{\prime}\right)$. We observe

that $\mathcal{L}_{c l}^{(\Gamma)}$ plays the role of the classical Liouville operator, with $\varphi^{n}$ replaced by $\left\langle\tilde{\varphi}^{n}\right\rangle$. The difference $\left\langle\tilde{\varphi}^{n}\right\rangle-\varphi^{n}$ is accounted for by the terms involving $G$. They induce a dependence of $\mathcal{L}$ on $\Gamma$ and turn the evolution equation non-linear. The quantum corrections are all proportional to $\left(\frac{\delta \Gamma}{\delta \pi}\right)^{3}$.

The evolution equation (23) has a fixed point $\Gamma_{*}(\beta)$ corresponding to thermal equilibrium where $\rho=Z_{0}^{-1} e^{-\beta H}, Z_{0}=\operatorname{Tr} e^{-\beta H}$ in Eq. (3). This can be computed by functional integral methods [3]. It is far from obvious, however, if and in what sense the solutions of Eq. (23) with non-equilibrium initial conditions approach this fixed point. A uniform approach is not possible due to the existence of infinitely many other fixed points which correspond to incoherent mixtures of eigenstates of $H$. At best, the equilibrium fixed point can be approached if we restrict the discussion to correlation functions of suitably averaged fields (coarse graining) or to other subsystems. Thermalization can also be achieved by a coupling to an environment, thus introducing a stochastic element in the equations of motion. A truncation of $\Gamma[\varphi, \pi, t]$ may destroy the existence of the infinitely many fixed points. Information about higher 1PI correlations or their precise momentum dependence is omitted in this way. It is conceivable that truncated equations have a more uniform approach to the fixed point than the exact ones. Good truncations should at least retain those terms that play an important role in the computation of $\Gamma_{*}(\beta)$ by the solution of renormalization group equations in dependence on a coarse graining scale [4]. A better understanding of the impact of truncations will be crucial for the practical use of the present formalism.

\section{References}

[1] C. Wetterich, hep-th/9612206, hep-th/9702125.

[2] R. Balescu, Equilibrium and Non-Equilibrium Statistical Mechanics, John Wiley (1975).

[3] J. Zinn-Justin, Quantum Field Theory and Critical Phenomena, Oxford University Press (1989).

[4] C. Wetterich, Nucl.Phys. B352 (1991) 529; Phys.Lett. 301B (1993) 90; Z.Phys. C60 (1993) 461.

\footnotetext{
${ }^{2}$ Note that $\Gamma^{(2)}$ has indices $(x, \gamma)$ and $\left(x^{\prime}, \gamma^{\prime}\right)$ with $\gamma=(\varphi, \pi)$.
} 